\title{
Chamanisme et transformation sociale à Touva
}

Shamanism and social transformation in Tuva

\section{Charles Stépanoff}

\section{(2) OpenEdition}

Journals

Édition électronique

URL : https://journals.openedition.org/emscat/487

DOI : 10.4000/emscat.487

ISSN : 2101-0013

Éditeur

Centre d'Etudes Mongoles \& Sibériennes / École Pratique des Hautes Études

Édition imprimée

Date de publication : 1 décembre 2004

Pagination : 155-183

ISBN : 2-9518888-2-1

ISSN : 0766-5075

Référence électronique

Charles Stépanoff, «Chamanisme et transformation sociale à Touva », Études mongoles et sibériennes, centrasiatiques et tibétaines [En ligne], 35 | 2004, mis en ligne le 17 mars 2009, consulté le 13 juillet 2021. URL : http://journals.openedition.org/emscat/487 ; DOI : https://doi.org/10.4000/emscat.487

Ce document a été généré automatiquement le 13 juillet 2021.

(c) Tous droits réservés 


\section{Chamanisme et transformation sociale à Touva}

Shamanism and social transformation in Tuva

Charles Stépanoff

\section{Kyzyl, capitale du chamanisme}

1 Après la disparition brutale du discours communiste imposé durant soixante ans par un régime qualifié aujourd'hui sans hésitation à Touva de totalitaire, puis le départ massif des Russes dans les années 1990, les Touvas se sont trouvés dans une situation qu'on pourrait qualifier de décolonisation culturelle, avec pour tâche urgente de reconstruire une idéologie nationale ${ }^{1}$. Les phénomènes qui se sont produits depuis la perestroïka sont riches, complexes et inattendus. Les profonds bouleversements sociaux survenus avec l'apparition de l'économie de marché n'expliquent pas tout dans les métamorphoses $\mathrm{du}$ chamanisme; le rôle des personnalités individuelles est considérable, non moins que celui des interventions étrangères. À vrai dire, la description des institutions chamaniques contemporaines à Touva semble quelquefois devoir faire le sujet d'un roman plutôt que celui d'une étude ethnographique, tant les facteurs psychologiques et les parcours individuels sont complexes.

2 Pendant la période soviétique, les pratiques chamaniques ayant échappé au contrôle des chamanes pour devenir le fait de profanes, la culture matérielle, pour des raisons de discrétion, s'était réduite à sa plus simple expression. Aujourd'hui les chamanes ont à peu près gagné la bataille de la reconquête du monopole des pratiques, et en premier lieu celle de la fabrication des êêren ${ }^{2}$. Dans cette culture matérielle renaissante, caractéristique du chamanisme touva contemporain, se reflètent les enjeux, les choix stratégiques, et même les solidarités personnelles qui forment la trame d'un phénomène social entièrement neuf, qui a pour nom «tradition ».

3 Sans aucun doute, c'est à l'introduction de l'organisation associative que le chamanisme touva doit son essor actuel. L'exercice solitaire de l'art chamanique n'est plus valable dans une société sédentarisée depuis quarante ans. En contexte urbain, il 
est nécessaire de se mettre en avant, de faire sa publicité, de recevoir une caution officielle, et seule l'union rend efficaces les efforts et les stratégies tendant vers ce but. Face à la concurrence traditionnelle du bouddhisme, le chamanisme, tel qu'il se présentait en 1991, avait peu de chances d'emporter des succès. Le gouvernement en quête de culture nationale préférait mettre en valeur le bouddhisme, prestigieux au niveau mondial et organisé de façon centralisée, plutôt que quelques chamanes isolés à la santé mentale douteuse. Le bouddhisme, de plus, offrait pour les subventions des débouchés concrets susceptibles de séduire le pouvoir local : ainsi la construction de xürêê (temples bouddhiques) qui s'inscrivait au premier plan d'une politique de reconquête du paysage urbain. Rien ne pouvait mieux que ces grands monuments donner un aspect local ou " national » à la capitale banalement soviétique qu'est Kyzyl. La tête de proue du chamanisme touva, M. B. Kenin-Lopsan, grâce à son habileté et son énergie autoritaire inépuisable, a su renverser cette situation. Il a su faire valoir sa caution de savant, et, en organisant la société Düngür en 1992, a permis au chamanisme d'apparaître au gouvernement comme un partenaire sérieux et pérenne. Ses efforts ont fait du chamanisme, sur le plan sociologique, une institution moderne adaptée aux réalités de la société contemporaine, et, sur le plan idéologique, un pan essentiel de la culture touva que le gouvernement ne saurait plus se dispenser de soutenir.

Notre description ne pourra manquer d'évoquer l'aspect économique du renouveau chamanique, au risque de paraitre adopter sur un phénomène essentiellement «spirituel» un point de vue teinté de cynisme. Mais jeter un voile sur cette réalité, comme le font trop souvent les Occidentaux mystiques ou savants dans leurs récits de voyage à Touva, est d'autant moins justifié que le sujet revient fréquemment dans la bouche des clients, mais aussi des chamanes eux-mêmes qui n'éprouvent nulle pudeur à l'aborder. Le caractère marchand de leur activité est revendiqué par les chamanes qui offrent, à leurs yeux, un service réel et efficace à leurs clients. Tant il est vrai que le chamanisme à Kyzyl, en tant qu'institution sociale, n'est pas à considérer comme la survivance d'une tradition ancienne au milieu de la société de marché, mais plutôt comme un produit original de celle-ci. Certes, les stratégies de légitimation symbolique des chamanes font souvent appel à la tradition, et leurs pratiques rituelles témoignent de ce besoin d'enracinement. Mais la tradition invoquée, connue par les livres, les récits ou, plus rarement, l'observation dans un contexte rural, n'a jamais sa source dans des faits kyzyliens. D'un point de vue purement sociologique, le chamanisme urbain est un phénomène original sans précédent dans l'histoire de Touva.

5 Les visiteurs étrangers en général, et les ethnologues en particulier, ont acquis un rôle tout à fait considérable dans le mode de légitimation mais aussi de financement du chamanisme touva. Le voyageur en vacances peut éventuellement fermer les yeux sur l'aspect organisé de sa réception par les indigènes, afin de maintenir pour son plaisir l'illusion de l'aventure, de la découverte et du "premier contact». Mais l'ethnologue peut difficilement ignorer l'attention pressante et répétée dont il fait l'objet. Loin d'être un découvreur qui va révéler au monde des faits témoignant dans leur pureté d'un système de pensée original et cohérent, une "culture ", où les individus ne sont que les représentants innocents et intacts, les produits, d'une identité collective, l'ethnologue est aujourd'hui dans la société touva un personnage attendu et connu, dont l'identité varie mais dont la fonction est rigoureusement instrumentalisée. Faire de l'ethnologie à Touva, c'est toujours arriver après un ethnologue canadien ou américain, une sociologue italienne, un ethnomusicologue britannique, - non pas dix 
ans après, mais tout au plus une semaine ou, situation sans doute la plus pénible mais dont les chamanes savent jouer, en même temps.

6 Les chamanes sont prêts à rendre certains services utiles pour la carrière académique de l'ethnologue, laquelle a peu de secrets pour eux, en échange de quoi ils attendent un soutien financier et symbolique. Qu'il le veuille ou non, l'ethnologue qui accepte de payer son informateur ne fait pas qu'entrer dans un système économique, il l'entretient, - l'acceptant aux yeux des clients locaux, il le définit comme acceptable. Quant à ne pas payer, il ne faut pas y songer à Kyzyl : les tarifs pour étrangers sont affichés au mur. Quand il fréquente tel ou tel chamane, notant sa parole en l'approuvant complaisamment, déguisant son scepticisme en admiration, photographiant le maître, le filmant, il contribue à la sacralisation de sa parole et à la légitimation de son statut. Bien plus, en tant qu'Occidental qui vient d'accomplir spécialement un voyage immense pour ce chamane, il lui confère un prestige inouï dont son interlocuteur saura remarquablement bien user auprès de sa clientèle.

7 La complexité des faits auxquels il est confronté et le rôle d'acteur qu'il est amené à jouer mettent l'observateur face à des questions nouvelles sur sa position, qu'il ne peut, sans inconscience, éluder. S'il ne veut pas se contenter de jouer, après bien d'autres, le rôle que les indigènes lui ont soigneusement préparé, il devra remettre en cause l'emploi de certains instruments traditionnels correspondant à une vision plus fixiste que dynamique des faits et des pratiques culturels. Analyser la réalité contemporaine du chamanisme touva à travers la problématique de la « culture d'une société », c'est se contraindre à lire les pratiques comme des survivances d'une identité conservée contre vents et marées, d'une essence infrangible, c'est reconnaître une continuité que soixante ans d'athéisme d'État ont détruite, c'est en fin de compte prendre comme naturel ce que les acteurs s'efforcent justement de fonder en nature : la pérennité de la " touvinité ", l'éternité du chamanisme. Ne regarder que le résultat ou le but visé par le travail des acteurs, c'est ne pas voir ce travail. C'est manquer la part de réinvention, d'adaptation et d'imagination qu'il y a dans la « renaissance » d'une identité culturelle ; c'est aussi s'interdire d'observer les stratégies ambiguës et complexes des acteurs, pour qui, dans le contexte d'une société caractérisée par le bilinguisme, l'interpénétration et la concurrence des cultures et des systèmes de pensée, le fait d'être chamane, chamaniste ou tout simplement Touva (plutôt que bouddhiste, athée, évangéliste, Russe) n'est pas une fatalité de nature mais un choix délibéré, une revendication.

8 La prise de conscience de ces problèmes permettra du moins à l'ethnologue de limiter l'étendue de sa surprise face à son portrait, lorsqu'il se trouvera un jour ou l'autre décrit dans une étude ethnographique locale comme un acteur essentiel de son " terrain », comme le rédempteur de la culture qu'il étudie, - c'est-à-dire le démiurge de son propre objet d'étude, et en définitive peut-être le mythologue de ses propres fantasmes.

9 Le nombre de chamanes à Kyzyl est devenu considérable, tout le monde le reconnait, y compris les chamanes eux-mêmes. Cette situation ne réjouit guère la population, car l'abondance de l'offre ne provoque pas de baisse des tarifs, au contraire. La situation apparente de marché, où les sociétés constitueraient une offre en libre concurrence, n'est qu'une première impression qui laisse place bientôt, à mesure que l'enquête s'approfondit, à un tout autre tableau où se dessinent des réseaux complexes de dépendance, de solidarité et de féodalisme. Deux sociétés dont les membres se présentent comme absolument indépendants s'assemblent le soir pour une cérémonie 
commune ou un congrès, sur l'ordre de Kenin-Lopsan ${ }^{3}$ qui n'appartient ni à l'une ni à l'autre, mais dont l'autorité morale transcende les différentes chapelles. Cependant, à mesure que les langues se délient, ce tableau hiérarchisé lui-même se modifie : la belle organisation pyramidale régulant le transfert de la légitimité du haut vers le bas apparaît comme une composition bien arrangée. Verticales et horizontales, des tensions existent bel et bien, même si elles ne s'organisent pas sous la forme d'un marché régi par la libre concurrence. Il s'agit plus de guerres larvées, dont on verra qu'elles jouent un rôle essentiel dans le fonctionnement du chamanisme contemporain à Kyzyl, et dans les représentations du commerce avec la chance et le malheur.

Nouvellement arrivé de l'aéroport de Kyzyl ou, s'il a emprunté la voie royale du chemin de fer Moscou-Abakan, conduit en taxi depuis la Khakassie, le voyageur se trouve vite confronté aux sociétés chamaniques. Elles sont quatre à Kyzyl : Düngür, Tos-Deer, XotigTajga, et Adyg-Êêren. La société Xotig-Tajga est la seule à être totalement indépendante de Kenin-Lopsan qui la considère comme une association de malfaiteurs et de charlatans. Elle est présidée par Sajlyk-Ool Kanxyr-Ool, co-fondateur avec KeninLopsan de Düngür. La dispute entre les deux anciens amis alla jusqu'à donner lieu à une affaire en justice. Preuve de l'ostracisme efficace dont elle est victime, la société XotigTajga est la seule à ne pas être citée dans le guide touristique qu'emportent les visiteurs russes à Touva, Le Petit Futé. N'ayant pas visité cette société, nous ne la décrirons pas.

Souvent le touriste étranger ne connaît rien de ces sociétés, ne s'attendant nullement à leur existence ; elles, au contraire, le connaissent bien, lui, ses attentes, ses rêves, mais aussi ses moyens financiers. Un touriste mystique, même s'il est un étudiant tchèque désargenté en fin de tour du monde, est immédiatement rangé dans une catégorie d'humains infiniment plus riches que les Touvas, - pour pénible qu'elle soit au visiteur, cette assimilation n'en est pas pour autant injustifiée, Touva étant la république la plus pauvre de la Fédération de Russie. Le touriste rend visite d'ordinaire en premier lieu à la société Tos-Deer (les Neuf Ciels), guidé par la recommandation du Petit Futé russe : "c'est l'endroit où les chamanes reçoivent le public " (les autres sociétés n'ont pas droit à une telle publicité) ou par quelques mots du guide Lonely Planet, qui signalent la présence de cette institution pittoresque sur la rive gauche du Iénisséi.

\section{Tos-Deer (les Neuf Ciels)}

12 La société Tos-Deer est présidée par l'une des plus fameuses chamanes de Touva, la redoutée Aj-Čürek ${ }^{5}$. Non loin du monument symbolisant le Centre de l'Asie que des géographes anglais ont localisé au xIX ${ }^{e}$ siècle en un lieu qui allait devenir plus tard le centre de la ville de Kyzyl, sur une large promenade longeant la rive du Iénisséï, on remarque de loin les installations de la société Tos-Deer. Devant une maison de bois sans étage, deux yourtes sont dressées sur le gazon, ce qu'on ne voit jamais dans les jardins de Kyzyl (contrairement aux villages de province). Mais ce qui frappe le plus, c'est une grande construction composée de neufs poteaux plantés dans des tas de pierre, et reliés entre eux par des ficelles, où sont suspendues des masses de rubans rituels (čalama) de toutes les couleurs.

Le touriste tenté de photographier est abordé par l'un des gardiens de la société, qui lui signale qu'il ne peut le faire sans autorisation des chamanes, et de leur présidente, Ajčürek. On fait pénétrer l'étranger dans la petite maison et, si les chamanes sont en mission à l'extérieur, ce qui est fréquent, il lui faut patienter dans la salle d'attente où il 
obtiendra des renseignements de la caissière et des affiches placées au mur. Les tarifs pour étrangers se divisent en trois catégories par ordre croissant : citoyens russes (les Russes «d'au-delà des Saïan » sont considérés à Touva comme des étrangers), autres citoyens étrangers, et professionnels. Le droit de photographier une séance chamanique s'élève à 800 roubles pour un étranger, pour un film documentaire la somme à verser est de 3000 roubles. Mais même le paiement immédiat des droits sans marchandage ne permettrait pas à l'étranger de photographier ce qu'il désire. Aj-Čürek ne reçoit aucun " hôte » sans l'avoir fait passer par le cabinet de M. B. Kenin-Lopsan pour qu'il obtienne de celui-ci une lettre de recommandation.

Le cabinet de Kenin-Lopsan se situe dans la cour intérieure du musée de Kyzyl ${ }^{6}$. L'accès se fait par la caisse du musée où un panneau annonce le tarif d'une rencontre avec le grand homme. Tout visiteur s'intéressant au chamanisme touva doit connaître l'épreuve d'une première rencontre avec Kenin-Lopsan, dont il garde en général un pénible souvenir. L'autorité de Kenin-Lopsan trouve historiquement sa source non à Touva mais dans la reconnaissance d'institutions étrangères. Les deux titres qu'il décline et que ses disciples nomment à son sujet sont celui de "docteur en histoire " qui lui a été conféré par l'université de Léningrad et celui de "Trésor vivant du chamanisme » dont l'a honoré en 1994 le Fonds américain pour les études chamaniques. Grâce à sa double réputation de principal anthropologue local (encore que ses travaux personnels concernent surtout le folklore) et de puissant chamane, Kenin-Lopsan est arrivé à concentrer entre ses mains un pouvoir et un prestige exceptionnels, qui font de lui la principale autorité morale du pays. Sa pratique chamanique est cependant limitée à la divination par les pierres (xuanak) et à la prestation de conseils. Il n'est pas rare que des Touvas se rendent chez lui pour lui demander quel nom donner à leur enfant nouveau-né. Des enfants des écoles auxquels il rend de terrifiantes visites, jusqu'aux chamanes nommés par lui, en passant par l'intelligentsia et les pouvoirs politiques, tous manifestent pour lui une vénération mêlée de peur, et aussi quelquefois de secrète ironie. Seuls quelques libres-penseurs parviennent à se soustraire franchement à la terreur qu'il impose universellement.

Le voyageur mal informé se rend auprès de lui les mains vides, fréquente erreur à laquelle Kenin-Lopsan répond par un important discours sur l'avarice des étrangers, les mœurs "païennes » des Touvas et la nécessité d'offrir des cadeaux à ces derniers. Si l'étranger est assez habile, Kenin-Lopsan passe ensuite à sa machine à écrire où il tape une fiche d'identité rassemblant les informations concernant le visiteur. Puis il entame l'écriture d'une lettre de recommandation destinée à " ses chamanes ", qui les autorise à recevoir l'étranger et à lui faire connaître leurs secrets. Muni de cette lettre, l'étranger pourra revenir voir Aj-Čürek et s'entendre avec elle.

Quelle est la part de l'aspect économique dans les relations de soumission de Tos-Deer envers Kenin-Lopsan ? L'insistance que Kenin-Lopsan met à ce que l'étranger conclue un accord avec Tos-Deer, et l'expression dont il qualifie l'étranger s'il tarde à le faire (žadnyj, en russe "avide») semble indiquer que cette part existe dans une certaine proportion, ce que plusieurs informateurs nous ont confirmé. Au total, les sommes versées par les étrangers peuvent être importantes, car les services proposés par TosDeer sont nombreux. Connaissant le goût des "hôtes" pour la couleur locale, les chamanes ont fait installer près de leur centre des yourtes où ils peuvent séjourner tout au long de leur stage chamanique. La société Tos-Deer reçoit des étrangers pour de longues durées et peut leur offrir un ensemble de propositions incluant participation à 
toutes les cérémonies suivies d'explications personnalisées avec interprète, enseignement chamanique, séjour dans un campement nomade chez la famille de l'un des chamanes, visites des curiosités naturelles de la république (sources sacrées aržaan, lacs salés) et rituels dans leurs environs.

17 C'est en grande partie à la personnalité de la chamane Natacha que la société Tos-Deer doit son succès auprès des étrangers. En comparaison, les autres sociétés restent loin à l'écart. Natacha, après des études de psychologie à Moscou, est venue s'installer à Kyzyl, et elle est actuellement l'adjointe d'Aj-Čürek, la remplaçant lors de ses voyages à l'étranger. Elle parle l'anglais ce qui lui permet d'assurer la communication avec les étrangers, mais elle ne connaît pas le touva. Comme chamane, elle reçoit une certaine clientèle locale, même si on ne lui prête pas la réputation d'être particulièrement puissante.

18 Aujourd'hui la société Tos-Deer semble être victime de son succès auprès des étrangers. La population locale est rebutée par les tarifs élevés des services, et par l'attitude fière des chamanes. Plusieurs observateurs ont confirmé notre impression que la clientèle de Tos-Deer a fortement chuté entre 2002 et 2003.

19 En revanche, Tos-Deer établit fermement son influence sur la ville grâce à des cérémonies publiques nocturnes qui ont lieu tous les mois à la pleine lune. Plusieurs dizaines de personnes, jusqu'à une centaine, se rassemblent autour d'un bûcher installé face au local de la société. L'assistance est essentiellement composée de Touvas qui prient les mains jointes à la manière bouddhique, mais aussi d'étrangers, hôtes de TosDeer, simples visiteurs, ou journalistes. Autour du foyer, les chamanes battent du tambour ensemble. La cérémonie est clôturée par un discours d'Aj-Čürek qui, sur un ton véhément, fait le récit des dernières œuvres de la société Tos-Deer, et recommande aux Touvas de vivre dans le respect des traditions chamaniques.Ces cérémonies sont l'exclusivité des chamanes de Tos-Deer et sont condamnées par tous les autres chamanes de la ville. La cause de cette désapprobation unanime, outre une jalousie compréhensible, réside dans le fait de frapper du tambour collectivement. Pour la plupart des chamanes contemporains, la séance chamanique doit obligatoirement être menée par un chamane seul. Pourtant dans la littérature on signale certains cas, rares certes, où deux chamanes pouvaient chamaniser ensemble ${ }^{7}$. Selon Vajnštejn (1991, p. 253), l'investiture du chamane touva comprenait même obligatoirement cinq nuits de séance chamanique à deux avec un chamane parrain. Ce rite était nommé xam baštar ( l'enseignement du chamane »).

20 En août 2003, la cérémonie de la pleine lune fut particulièrement importante en raison du symposium international sur le chamanisme touva, organisé par Kenin-Lopsan dix ans après la première réunion de ce type en 1993. Les invités autrichiens du colloque, membres de la filiale viennoise du Fonds américain pour les études chamaniques, se considérant eux-mêmes comme chamanes, prirent part à la séance chamanique. Cet " événement » reçut un écho mondial en raison de la présence d'une équipe de l'agence de presse Reuters, dont les images ont été depuis vendues dans le monde entier. On comprend le mécontentement des autres sociétés voyant le monopole que Tos-Deer avait réussi à établir sur les visiteurs étrangers. Auparavant, sur ordre de KeninLopsan, toutes les sociétés avaient envoyé leurs chamanes en costume pour former un impressionnant comité d'accueil à l'aéroport, puis à nouveau au musée. Or les visiteurs, au cours de leur séjour, fréquentèrent exclusivement la société Tos-Deer, lui réservant le prestige et les bénéfices matériels liés à la réception d'étrangers. D'après les autres 
sociétés, il faut voir dans cette préférence non pas une initiative des étrangers, mais une instruction de Kenin-Lopsan en faveur de la société qui lui est la plus fidèle.

\section{Düngür (le Tambour chamanique)}

21 La société Düngür est la plus ancienne de Touva. Elle a été fondée en 1992 par KeninLopsan et a été déclarée comme association religieuse en 1997. Son local se situe rue Rabočaja, comme la société Adyg-Êêren, mais nettement plus près du centre-ville, à quelques centaines de mètres du marché couvert. L'entrée du local se signale de la rue par deux poteaux ornés de quelques rubans. L'effet est nettement plus modeste qu'à Tos-Deer.

Un incident grave est survenu en 2000 qui condamne désormais Düngür à une mise à l'ombre irréversible. Tout en tenant à garder le contrôle moral sur Düngür, KeninLopsan souhaitait se soulager de la tâche administrative et préparer sa succession, aussi nomma-t-il un de ses disciples, Kara-Ool Dončun-Ool, président de l'association. Ce poste administratif ne devait pas avoir en principe de signification réelle, cependant Dončun-Ool le prit très au sérieux et tenta de gagner son indépendance par rapport au fondateur. Inévitablement un conflit se produisit entre les deux hommes, et KeninLopsan démit le président de ses fonctions sous un prétexte qui paraît aujourd'hui peu vraisemblable (alcoolisme).

Kenin-Lopsan donna dès lors sa faveur à la société Tos-Deer. Bien que soumise, la société Düngür est toujours considérée comme légèrement rebelle, et, de fait, elle est aujourd'hui plus indépendante du contrôle de Kenin-Lopsan que ne l'est Tos-Deer. Officiellement le président actuel de Düngür, Dugar Surun, reconnaît l'autorité du fondateur Kenin-Lopsan et rend hommage à son œuvre, cependant aucune coopération, aucun flux d'étrangers (à la fois biens économiques et biens de prestige) ne viennent plus récompenser cette soumission.

Pendant le symposium d'août 2003, une banderole était tendue entre les deux poteaux de l'entrée avec cette inscription: «Bienvenue aux membres du symposium sur le chamanisme!», successivement en touva, en anglais et en russe. Mais cette initiative ne suffit pas pour attirer les étrangers, que Kenin-Lopsan avait réservés à Tos-Deer.

Düngür, tombée dans la défaveur de son fondateur, et incapable de rompre avec lui pour s'engager dans une dynamique nouvelle, est aujourd'hui en perte de vitesse. Elle ne peut que subir les rétorsions de Kenin-Lopsan, sans avoir assez d'autonomie pour y résister, par exemple en attirant des étrangers directement à elle. Quant à son ancien président rebelle, Dončun-Ool, il a décidé de prendre le risque de l'indépendance en créant la société Adyg-Êêren.

\section{Adyg-Êêren (l'Êêren-Ours)}

La société Adyg-Êêren est la plus prometteuse d'avenir. Elle a su capter la faveur des pouvoirs publics en prenant un caractère national que les autres, cantonnées à l'environnement kyzylien, n'avaient pas pu atteindre. Comme souvent, cette société doit beaucoup à la personnalité de son fondateur. Dončun-Ool travailla d'abord comme employé d'une firme de production de fourrures, l' «or brun» dont Touva est particulièrement riche. De cette première époque, il conserve quelques bêtes 
empaillées, qui ont trouvé plus tard une nouvelle vie comme êêren dans sa société chamanique. Kara-Ool Dončun-Ool travailla ensuite au musée des Soixante-Héros, comme chauffeur de Kenin-Lopsan. Il l'a accompagné dans ses expéditions savantes, au cours desquelles ils rapportèrent des stèles turques anciennes, mais aussi quelques objets chamaniques, actuellement conservés dans les collections du musée. KeninLopsan décela un don chamanique en Dončun-Ool, petit-fils comme lui d'une chamane persécutée par les autorités soviétiques, et il lui enseigna ses connaissances. Une fois devenu chamane, Dončun-Ool sauva plusieurs fois Kenin-Lopsan de la maladie en reculant le terme de sa vie. Kenin-Lopsan lui confia en 2000 la direction de Düngür et lui remit le titre de "Grand Chamane » (ulug xam). Ce titre existait traditionnellement : il est signalé par Vajnštejn (1961, p. 182) chez les Todjes. Suivant son goût pour la chose administrative, Kenin-Lopsan fit imprimer spécialement une carte de «Grand chamane » pour Dončun-Ool, avec le numéro 001. Dončun-Ool affirme qu'aucun autre chamane n'a reçu depuis cette distinction.

Comme on l'a vu, Dončun-Ool se montra peu reconnaissant envers son maître et fut bientôt démis de ses fonctions. Le révolté gagna alors à sa cause plusieurs chamanes qui avaient subi les brimades de Kenin-Lopsan, parvint à réunir un groupe important qui rejeta de facto l'autorité du savant et créa en 2001, sans son autorisation, une société indépendante, Adyg-Êêren. Le "schisme" (puisque Dončun-Ool nomme ainsi cet événement, dont Kenin-Lopsan cherche au contraire à réduire l'importance) était consommé.

L'association ayant atteint le nombre de vingt-six chamanes, elle est aujourd'hui la plus nombreuse de Touva. Dončun-Ool a reçu du gouvernement en janvier 2003 une belle automobile Volga,qui lui permet de se déplacer en ville et en province pour répondre aux commandes de ses clients. Faveur plus importante encore, il a obtenu du gouvernement un très grand terrain avec plusieurs corps de bâtiments à l'abandon. C'est là qu'il installera son futur centre chamanique international qui s'ajoutera à l'actuel petit local de la rue Rabočaja. Ce centre, s'il voit le jour, sera le plus grand de Touva, et sans doute de toute la Russie. Les travaux sont déjà en cours, et Dončun-Ool s'est installé avec sa femme dans une pièce de l'un des bâtiments déserts.

Il est intéressant de noter qu'une société aussi hostile à Tos-Deer et Düngür que l'est Adyg-Êeren et qui fait entendre de sévères condamnations à l'égard de tout ce qui en vient, leur fait cependant des emprunts directs en matière de stratégie commerciale. Ainsi, l'installation de yourtes pour recevoir les étrangers fait partie des projets de Dončun-Ool. Mais son esprit entreprenant a donné au concept une bien autre ampleur que celle dont se satisfont les sociétés concurrentes. Il a déjà commandé huit yourtes, dont six seront installées dans le futur centre, tandis que les deux dernières rejoindront la cour de l'ancien local. Le centre sera de plus équipé d'une connexion au réseau internet à l'usage des visiteurs étrangers.

Tous ces projets seraient vains si la venue d'une clientèle étrangère n'était pas garantie, et si les chamanes de la société ne disposaient pas d'un prestige suffisant dans la population locale. Mais précisément, Dončun-Ool est resté suffisamment longtemps dans le giron de Kenin-Lopsan pour tirer de lui le prestige maximal (le titre de Grand Chamane) ainsi que des relations amicales avec de nombreux étrangers. Les plus dévoués d'entre eux lui servent de relais à l'étranger dans sa quête de soutiens financiers et contribuent à sa publicité. L'un d'eux, l'Anglais Ken Hyder, a ainsi créé sur son site internet une page ${ }^{8}$ consacrée à Dončun-Ool, où l'on apprend que celui-ci est 
"l'un des chamanes les plus puissants et les plus efficaces de son pays », qu'« il est respecté comme guérisseur, s'attaquant aux cas les plus variés, y compris le cancer », et qu'il « veut voyager en Occident, principalement pour soigner, mais aussi pour donner des conférences et présenter les techniques qu'il utilise ». L'adresse e-mail et les coordonnées postales de la société sont données à la fin de la page à l'attention des visiteurs qui souhaiteraient inviter le chamane.

31 Adyg-Êêren a su prendre son indépendance par rapport à Kenin-Lopsan, et a pu ainsi créer ses propres réseaux de solidarité national et international. Elle a pu notamment nouer des liens directs avec les autorités gouvernementales. Cependant, des réconciliations périodiques et des actes d'allégeance purement symboliques envers Kenin-Lopsan lui ont permis d'éviter une condamnation officielle de celui-ci et l'ostracisme qui en aurait découlé, comme celui dont souffre encore la société félonne Xotig-Tajga. C'est cette politique habile menée par Dončun-Ool qui vaut à sa société sa prospérité actuelle.

\section{Les relations entre les sociétés : solidarité, trahisons, complémentarité}

32 L'institutionnalisation du chamanisme a entraîné une standardisation du rituel. Les chamanes dirigeants des sociétés Tos-Deer et Adyg-Êêren sont d'anciens membres de Düngür et donc d'anciens élèves de Kenin-Lopsan; c'est là qu'ils ont appris le fonctionnement d'une société chamanique et les tarifs des différents services. Auparavant, dans le chamanisme traditionnel, l'ordre de grandeur de la rétribution d'une séance de purification ou de soin n'était pas déterminé précisément. On pouvait donner une écharpe de soie, un mouton, ou un cheval. Le système de rétribution était fondé sur l'échange plutôt que sur l'achat. Déterminer au rouble près la valeur d'une divination, voilà ce qu'a accompli d'essentiel la société Düngür au début des années 1990, sous l'impulsion de Kenin-Lopsan, et inspirée par des modèles divers: le bouddhisme, mais aussi les néo-chamanes américains de Michael Harner. Le tarif fixe a donné un ordre de grandeur qui a permis de transformer le rite chamanique en un service introduit dans un système de vente. Dès lors, nécessairement, le service chamanique voyant sa rétribution uniformisée devait se standardiser lui-même. Pour la même somme, le même service devait être offert, ni plus afin de ne pas brader le rite, ni moins afin de ne pas mécontenter le client qui a pu le voir accompli sur d'autres patients ou sur lui-même en une autre occasion. Autre conséquence importante, le rite n'appartient plus à un chamane en particulier, et n'est plus défini comme répondant à un problème précis. Le client vient pour une "purification " standard, quel que soit le chamane qui l'accomplit. Le rite n'est plus une réponse adaptée à une infortune spéciale, il est un produit marchand interchangeable. Le client vient obtenir un service déterminé quand il se rend dans le centre chamanique, alors qu'autrefois, c'était le chamane qui se déplaçait pour plusieurs jours à l'aal («campement») du malade, l'examinait et décidait de la solution la mieux adaptée à son cas. Bien souvent, cette solution était la fabrication d'un êêren particulier, dont l'identité et la forme dépendaient de l'histoire et des symptômes du patient. Dans une société chamanique de type kyzylien, les problèmes présentés par les clients sont divers, mais les réponses uniformes. Le flux continuel des clients à traiter, et donc le manque de temps, la présence des autres chamanes dans la même pièce, la standardisation et la 
marchandisation du service, tout cela tend à limiter l'initiative personnelle du chamane et l'originalité dans la recherche de la solution. Une réponse non standard serait immédiatement commentée par les autres chamanes, et sans doute critiquée par certains d'entre eux.

Les paroles des algyš («chants chamaniques») sont obligatoirement improvisées, mais d'un chamane à l'autre les mélodies et les invocations se ressemblent; quant à la durée, elle ne dépasse pas le quart d'heure alors qu'un rite chamanique se prolongeait autrefois souvent toute une nuit. Si les chamanes ont une part de liberté, c'est surtout en ce qui concerne le style qu'ils apportent au rite. Mais la structure du rite est prédéterminée et inchangeable. C'est le chamane-maître qui enseigne aux chamanes qu'il a recrutés la manière de l'accomplir correctement. Bien plus, il fait peser inévitablement son contrôle sur l'exécution du rite tout au long de la carrière du chamane qui devient son collègue de travail. Cette surveillance passive due à la promiscuité peut prendre une forme active : ainsi, il nous est arrivé, dans la société Adyg-Êêren, de voir le fondateur Dončun-Ool reprendre de façon humiliante un chamane âgé qui faisait une purification de façon non correcte. Devant une famille réunie, Dončun-Ool lui fit recommencer trois fois de suite le geste consistant à chasser la malédiction ou le mauvais esprit.

Les sociétés chamaniques sont regroupées autour d'un chef charismatique, à la fois guide spirituel et maitre des chamanes qui l'ont suivi. On aurait tort cependant d'en conclure qu'il existe autant de chamanismes à Touva qu'il y a de sociétés. À Touva, les schismes sont nombreux, mais les hérésies n'existent pas. Les diverses sociétés ne sont que les branches d'un même arbre, et quand on les descend, elles ramènent toutes au tronc commun qu'est la personne et l'œuvre de M. B. Kenin-Lopsan. Les divisions, plus ou moins graves et définitives, qui se sont produites depuis la réapparition officielle du chamanisme n'ont pas eu de cause idéologique, ni même de justification officielle de ce genre. Les raisons avancées par les acteurs sont des plus triviales : jalousies, rejet de l'autorité, partage inégal des revenus, harcèlements, abus sexuels. Mais, faute d'alternative, tous les chamanes restent, après leur séparations, fidèles à l'enseignement de celui qui les a investis, Kenin-Lopsan.

Cette uniformité de fait au niveau idéologique et rituel facilite les efforts de KeninLopsan pour cacher les divisions au public surtout étranger, et constituer une unité institutionnelle de façade. Selon lui, aucun chamane ne chamanise à Touva sans son accord personnel. Les scissions, purement contingentes, doivent être subsumées par son autorité qui demeure, elle, intangible. Cette situation de domination est stratégique, car elle autorise Kenin-Lopsan à se poser en intermédiaire entre le chamanisme touva et le monde extérieur. Il reçoit ainsi l'intégralité de l'aide du Fonds pour les études chamaniques de l'Américain Michael Harner et de sa filiale autrichienne, dirigée par Paul Uccusic. Lors du symposium du chamanisme d'août 2003, Kenin-Lopsan fit inviter la société Adyg-Êêren et la mit à l'honneur malgré leurs mauvaises relations, ce qui eut de quoi surprendre. En présence de la délégation autrichienne, Dončun-Ool fit un vibrant discours d'allégeance envers Kenin-Lopsan, qui dans ses hyperboles laissait loin derrière lui celui de la présidente de Tos-Deer. Quelques jours plus tard, il reçut une récompense officielle pour son œuvre de chamane. Il s'agissait d'un simple diplôme sans dotation, ce qui irrita fortement Dončun-Ool. S'estimant floués, lui et ses chamanes refusèrent de participer à la suite du symposium. En effet, ils ne reçurent aucune part de l'aide apportée par les délégations étrangères, 
n'ayant été invités que pour donner l'illusion d'un climat serein et d'une bonne entente entre les sociétés kyzyliennes. L'aide fut réservée, semble-t-il, pour l'essentiel à la société Tos-Deer, ainsi récompensée d'une fidélité indéfectible qui ne se réduit pas à de beaux discours mais se confirme chaque jour par une coopération loyale dans l'échange des étrangers, et sait aussi à l'occasion se mettre en scène : lors du symposium tous les chamanes de Tos-Deer défilèrent en costume au milieu du public en portant en triomphe le dernier ouvrage de Kenin-Lopsan, Mify tuvinskix šamanov.

Derrière l'entente cordiale présentée en vitrine pour les étrangers et la télévision, les tensions et les rivalités restent grandes entre les sociétés, donnant même lieu à des pratiques de sorcellerie, ou du moins à des rumeurs puissantes à ce sujet. La rivalité qui caractérisait traditionnellement les relations entre les chamanes n'a pas disparu mais s'est déplacée à un niveau supérieur, celui des sociétés. À tel point que les mauvais sorts (doora) sont devenus un élément essentiel du fonctionnement des sociétés chamaniques kyzyliennes. Les chamanes considèrent assez unanimement qu'aucune maladie n'apparaît sans avoir été causée par un doora. La séance de soin consiste donc à expulser du corps du malade le doora, souvent attribué à l'action nocive d'un chamane concurrent. De nombreux clients, sans même présenter de symptômes morbides, se présentent aux chamanes pour se faire retirer un doora. Un guignon tenace, un amour malheureux, le décès d'un proche, les persuadent d'en être victime et les plonge dans un état d'angoisse irrésistible. Moins bien protégés symboliquement par de puissants êêren que ne le sont les chamanes, ils se sentent les premières victimes de malédictions envoyées par des "chamanes noirs". L'état de guerre entre les sociétés est ainsi le moteur symbolique qui permet leur fonctionnement. Les êeren des chamanes sont considérés comme de véritables guerriers (le loup börü-êêren, l'ours adyg-êeren, ou la flèche $y d y k$-ok que Dončun-Ool compare à un missile sol-air), seuls capables de renvoyer à l'expéditeur la malédiction. Pour le client le remède sera, après la purification, la fabrication d'un petit protecteur, seul rempart possible contre la circulation des doora. Ce talisman est un simple fil dont une moitié est fixée à un êeren du local chamanique et l'autre au poignet du client. Ainsi, il se trouve soumis à la protection de l'êeren chamanique que le chamane a préalablement nourri. On observe que, de cette manière, le nourrissement de l'êêren reste l'exclusivité de son propriétaire, le chamane, bien qu'il puisse faire porter son pouvoir protecteur sur un tiers par l'intermédiaire d'un talisman. Le chamane peut également proposer la fabrication d'un êeren domestique qui sera installé dans l'appartement du client. L'êeren domestique se distingue de l'êeren chamanique en ce qu'il est incapable de nuire à un tiers.

En particulier, Dončun-Ool accuse régulièrement de sorcellerie Aj-Čürek, la présidente de Tos-Deer, réputée être une «chamane noire » (kara xam). Du point de vue de Düngür et d'Adyg-Êêren, la société Tos-Deer est essentiellement composée de chamanes noirs. Une des raisons de cette opinion est la forte présence d'étrangers : la jeune Natacha d'origine moscovite, un chamane khakasse et un chamane altaïen. Mais pour Düngür les activités d'Adyg-Êêren sont également maléfiques, et cette mauvaise opinion est réciproque. En généralisant, on peut dire que pour un chamane, tout chamane n'appartenant pas à sa société est potentiellement un chamane noir. La notion de kara xam est une notion relative: aucun chamane ne s'attribuerait à lui-même ce titre. Vajnštejn avoue qu'au cours de sa carrière, et parmi les dizaines de chamanes qu'il a interrogés, il n'est jamais parvenu à rencontrer un seul chamane noir, bien qu'il en ait souvent entendu parler (Vajnštejn 1991, p. 247). C'est que kara xam est une notion qui s'emploie exclusivement à l'égard des autres et non à son propre égard. On peut définir 
le chamane noir en disant que, du point de vue d'un chamane kyzylien quelconque, c'est un chamane ennemi, souvent rival, qui lui envoie des malédictions bientôt renvoyées à l'expéditeur, et dont les actions rituelles dangereuses ont des conséquences graves sur ses clients.

Notons enfin que ce système n'est pas fermé. Les chamanes kyzyliens ne sont pas les seuls accusés, mais aussi les chamanes et les sorciers " d'au-delà des Saïan », c'est-àdire les chamanes khakasses et les sorcières russes.

\section{Futilité de l'idéologie, importance du rite}

L'enseignement actuel proposé par les chamanes reflète pour l'essentiel les thèses développées par Kenin-Lopsan. Les propositions suivantes en offre un résumé :

Les Touvas sont des païens, héritiers authentiques d'une société archaïque. La patrie des Touvas est le Centre de l'Asie. Jusqu'en 1991, elle est restée isolée du reste $\mathrm{du}$ monde et pour cette raison l'état sauvage et brut de la société primitive s'est conservé ici dans son originalité

(Kenin-Lopsan 2000, p. 225)

L'idée essentielle est que le chamanisme est un trait fondamental de la "culture touva ", et que cette culture est liée à l'ethnie touva et à son territoire. Le chamanisme n'est donc pas une religion, mais plutôt une idéologie nationale. Les étrangers peuvent certes venir s'initier à Touva, mais c'est dans leur propres traditions nationales et auprès des divinités de leur lieu de naissance qu'ils devront trouver leur voie (ce que les touristes mystiques, en rupture de ban, sont peu disposés à admettre). En dehors de ce nationalisme religieux, les chamanes développent diverses thèses cosmologiques personnelles, en général peu soucieuses de cohérence. Du reste, ils exposent rarement ces idées, et les clients ne les interrogent guère à ce sujet (à la différence des visiteurs étrangers, en particulier des ethnologues). Il est notable que, dans sa société, DončunOol ne fasse peser aucun contrôle sur les discours formulés par ses chamanes en matière de cosmologie. Non qu'il n'ait pas d'idées en la matière, il en a de nombreuses, mais le sujet ne lui parait pas essentiel, pas plus qu'aux autres chamanes. Tout se passe comme si la cosmologie était, comme la philosophie transcendantale par exemple, quelque chose de profane aux yeux des chamanes, quelque chose de non sérieux.

Si les querelles dogmatiques ne sont pas la cause des schismes, c'est simplement parce qu'elles n'existent pas. Par contre, les désaccords au sujet des rites sont fréquents, et, s'ils ne sont pas la cause directe des querelles, ils alimentent du moins les tensions entre sociétés. Et sans doute est-ce là un trait essentiel du chamanisme que nous voyons réapparaître au cœur du sa variante kyzylienne contemporaine : la cosmologie n'est pas un domaine qui appartienne au contrôle du chamane, il s'en désintéresse. Son domaine est le rite, l'art du geste doué de puissance.

\section{Chamanes en province}

Les sociétés kyzyliennes ont étendu leur influence sur les différentes provinces de Touva, ce qui était immanquable en raison des liens très étroits unissant la capitale au monde rural. Bien que les urbains représentent officiellement $48 \%$ de la population, on peut dire qu'il n'y a pas de vrais « citadins » à Touva. La plupart des Kyzyliens ont des parents nomades, et rares sont les enfants qui n'ont jamais passé leurs vacances d'été 
sous la yourte. Les rites chamaniques, mais aussi les rites de mariage, exigent souvent d'avoir des liens avec le monde de l'élevage. La noce impose une grande dépense de viande fraîche qui est assurée par la famille de l'époux, tandis que la famille de l'épouse fournit les meubles. Un ingénieur au chômage peut sans difficulté se reconvertir dans l'élevage nomade, prenant simplement possession d'un troupeau dont il était propriétaire depuis la fête de sa première coupe de cheveux à trois ans (xalbyk tar) et dont son père ou son frère avaient jusque-là la charge.

Dans les villages où un ou deux chamanes se partagent la clientèle, le concept de réunion en société a naturellement peu d'emprise. On rencontre différents cas de figure. Un chamane de village peut être affilié à une société kyzylienne ; par exemple, il a pu se présenter à un président de société à Kyzyl, exposer les symptômes d'une maladie nerveuse, et, si celle-ci a été reconnue comme maladie chamanique, recevoir une formation de chamane. Dončun-Ool, après les avoir formés, renvoie de préférence ces chamanes dans leur province d'origine, conscient qu'il est de la surabondance dont Kyzyl est déjà victime, et désireux de donner une ampleur nationale à sa société. Les chamanes provinciaux « affiliés » sont ainsi titulaires d'une carte officielle de l'une des sociétés kyzyliennes et en tirent un prestige notable. Mais il existe aussi des sociétés locales; c'est le cas dans des villes importantes comme Chagonar. Ces sociétés sont organisées sur le modèle des sociétés kyzyliennes.

Plus remarquable, on trouve en province, assez couramment, des chamanes indépendants. Ces chamanes ne sont pas indépendants en raison d'un éloignement extrême qui les mettrait hors de portée de l'influence kyzylienne. (Cette influence ne connaît pas de limite à l'intérieur des frontières de la république de Touva, hormis peut-être à Todje, région très isolée et de culture différente.) Si les chamanes ne rejoignent pas d'eux-mêmes la capitale, on apprendra de toute façon leur existence et un membre de l'une des sociétés kyzyliennes viendra leur proposer d'adhérer, ou du moins d'accepter l'invitation d'un voyage à Kyzyl. Les chamanes locaux qui s'y refusent le font par modestie, ou par attachement à l'usage traditionnel selon lequel deux chamanes ne peuvent coexister l'un près de l'autre pacifiquement.

C'est le cas de Kim Nikolaïevitch Ondum, chamane d'Erzin et ardent défenseur du chamanisme indépendant. Une rencontre entre deux chamanes, rappelle-t-il fréquemment, doit aboutir nécessairement à un combat à mort. Lui-même, après un passage à la société Düngür où il a reçu l'enseignement de Kenin-Lopsan, a décidé d'exercer librement son art à Erzin. Kenin-Lopsan a naturellement considéré ce départ comme une trahison. D'autant plus que Kim Ondum a réussi à attirer l'attention de visiteurs étrangers. Erzin se trouve à l'extrémité de la route vers la Mongolie, au bord d'un lac de désert où les voyageurs viennent aboutir comme à un terminus. On a ainsi la surprise de découvrir Ondum, dans ce village misérable et reculé, en compagnie de disciples tchèques, finlandais ou italiens. Parmi eux une Italienne, reconnaissante d'avoir été guérie par Ondum d'une maladie pénible, l'a invité à faire un grand voyage en Europe. À cette occasion il participa en Suède à un colloque consacré au problème des minorités ethniques dans le monde. À son retour, Kenin-Lopsan reprocha à Ondum d'être parti à l'étranger sans son autorisation, ce à quoi il répondit: « Mais je ne suis pas ton fils! »C'est du moins ce que nous a raconté Ondum - version crédible si l'on en juge par l'état d'isolement au sein du chamanisme officiel dans lequel il se trouve malgré son aura internationale. Venu spécialement à Kyzyl pour le symposium sur le 
chamanisme d'août 2003, il fut sans doute repoussé, car on n'eut pas l'occasion de le voir une seule fois.

Leur indépendance, vue comme une absence d'accréditation, lèse les chamanes locaux non seulement auprès de la population locale, mais surtout, paradoxalement, auprès des autorités locales. Ces dernières ne se soucient pas de soutenir une variante régionale du chamanisme, qui ferait concurrence à celle de Kyzyl. Elles reconnaissent tout à fait le chamanisme kyzylien comme le seul légitime, pour la simple raison que les administrations qui les dominent n'en reconnaissent pas d'autre. Le prestige d'un chamane appartenant à une société reconnue et subventionnée par l'État, montré à la télévision auprès de Kenin-Lopsan, est naturellement infiniment supérieur à celui du chamane du village, chômeur, aperçu de temps en temps en état d'ébriété dans les rues, semblable aux autres habitants, et dépourvu de tambour. Ainsi, lorsque eut lieu à Mugur-Akcy, en mai 2002, une fête de consécration de l'aržaan du village accompagnée de concours de lutte traditionnelle (xüreš), le président du district, au lieu d'inviter l'un des trois chamanes du lieu qu'il connaissait parfaitement, préféra faire venir spécialement de Kyzyl, au prix d'un voyage de douze heures de piste, la célèbre chamane Aj-Čürek. Mais celle-ci ne vint pas, et la cérémonie eut finalement lieu sans chamane. Pour les chamanes kyzyliens, la présence d'un chamane local représente une concurrence à peu près négligeable. En août 2003, une affiche annonçait à la porte de l'hôtel du même village de Mugur-Akcy l'arrivée d'une chamane de la société kyzylienne réfractaire Xotig-Tajga. Sans aucune parenté dans le village, elle s'était installée à l'hôtel, accomplissait des rites dans la nature devant une petite assistance, et recevait des clients assez nombreux. Si le chamanisme indépendant de province soutient très difficilement la concurrence du chamanisme de type kyzylien, c'est qu'il lui manque une source de légitimation reconnaissable.

\section{Problèmes de légitimité : « Où sont les vrais chamanes?»}

47 La question du "vrai chamane » est une question qui revient très fréquemment dans les entretiens aussi bien avec les chamanes et leurs clients qu'avec les ethnologues locaux. L'inflation chamanique brutale de ces dernières années pose inévitablement le problème de la légitimité.

L'un des rôles très précieux que remplissent les sociétés, c'est celui d'instances de légitimation du statut de chamane. Autrefois, c'est le groupe (le campement aal) qui décidait si tel individu frappé par une maladie chamanique pouvait ou non devenir chamane. C'est en effet le groupe qui fournissait au chamane ses attributs, du costume au tambour. La situation n'est plus la même aujourd'hui: il n'y a pas en ville de communauté ayant des liens de solidarité suffisamment forts pour décider d'un commun accord de se munir d'un chamane, et faire l'effort de trouver une peau de bouquetin, des plumes d'aigle, etc., pour son tambour et sa coiffe. Devenir chamane résulte en ville essentiellement d'une initiative privée, il ne peut en être autrement. La laïcité du régime interdit aux pouvoirs publics, seule institution en mesure de jouer le rôle de la communauté traditionnelle, d'intervenir directement dans l'élection des chamanes. (Le président du gouvernement compte un chamane parmi ses conseillers, mais ce n'est pas lui qui l'a investi.) Or, si n'importe qui peut se faire chamane, si l'assentiment de la communauté et la réputation ne sont plus là pour fournir une 
garantie, alors il n'y a plus moyen de distinguer le «vrai chamane » du «charlatan ». Les premiers à subir les conséquences de cette crise sont les chamanes eux-mêmes: leur statut se trouve dénué de toute valeur si aucun organe n'en assure la légitimité. C'est pour cette raison qu'ils ont été amenés à créer eux-même cet organe manquant, en se rassemblant en sociétés. C'est M. B. Kenin-Lopsan qui, par son prestige de savant et d'écrivain, a pu donner sa légitimité à la première société chamanique, Düngür. Certains ethnologues locaux lui reprochent naturellement de se servir à des fins pratiques et personnelles de son autorité scientifique. En tous cas, la société Düngür a $\mathrm{pu}$, dès sa fondation, fournir à ses membres un certificat tangible de légitimité sous la forme d'une carte d'adhérent. C'est le fait d'être reconnu par ses pairs, eux-mêmes reconnus par Kenin-Lopsan dont le prestige est chaque jour confirmé par la télévision locale et les visites d'étrangers venus de loin, qui remplace désormais l'assentiment de la communauté.

On comprend les difficultés que rencontrent les chamanes isolés. Aux yeux de ceux qui ont adopté le système «kyzylien » de légitimation, leur existence ne peut être qu'une imposture. Seuls parviennent à se maintenir dans cette situation irrégulière des individus exceptionnellement talentueux, doués d'un charisme important, et qui par leurs seules prouesses sont parvenus à se construire une réputation. C'est le cas de Kim Ondum à Erzin qui représente un modèle alternatif à celui de Kyzyl. Il donne les preuves de son don chamanique par le récit des guérisons qu'il a accomplies, mais aussi par le résultat positif d'un test I.R.M. effectué en Europe sur son cerveau. Le test aurait prouvé scientifiquement ses particularités et son don. C'est un mode de légitimation de type individualiste fondé sur le talent personnel et l'exception.

Il faut reconnaître que le nouveau mode de production de la légitimité fourni par le modèle kyzylien est loin d'avoir convaincu la plupart des Touvas. Les chamanes montrent souvent leurs cartes devant un public qui semble indifférent. Les clients savent bien en général que la réunion des chamanes en société est contraire aux règles traditionnelles. Ils en parlent avec ironie, citant un proverbe touva qui dit : « Quand on n'a plus à manger, on se fait chamane. " Ils critiquent comme anormal ce nouveau mode de fonctionnement, ce qui ne les empêche pas de s'y soumettre. Une partie de l'intelligentsia académique, non impliquée dans le renouveau culturel touva, se montre plus franchement réfractaire quand on l'interroge : il s'agit surtout des ethnologues et historiens locaux. D'abord, les ethnologues du Centre des sciences humaines sont en général bouddhistes, comme c'est la règle traditionnellement pour l'élite sociale touva, et, de plus, il est vrai qu'ils connaissent mieux que les chamanes actuels le chamanisme traditionnel. À leurs yeux, l'existence des sociétés est illégitime, les pratiques qui y ont cours sont contraires aux règles relevées dans leurs travaux de terrain des années 1950-1960 auprès d'informateurs âgés. Plus d'une fois, on nous conseilla de nous pencher sur les notes de ces enquêtes non encore publiées plutôt que de fréquenter les sociétés. Ces personnes considèrent les chamanes kyzyliens comme des "charlatans " et certaines se risquent à critiquer l'attitude de Kenin-Lopsan. Les ethnologues touvas, quelquefois plus touvas qu'ethnologues, semblent tenir sans exception pour valable la distinction entre «vrai » et «faux » chamane. On peut résumer leur représentation du vrai chamane en disant qu'il vit très reculé dans un campement de nomades (par exemple en Mongolie), qu'il respecte scrupuleusement les règles rituelles comme celle de la combustion du genévrier, qu'il n'a subi aucune influence du bouddhisme, qu'il possède des dons d'extra-lucidité, et que de lui émane un puissant prestige immédiatement reconnaissable. Enfin, dernière et regrettable caractéristique, il est 
mort ou sur le point de mourir. Il est intéressant de noter que ce modèle est assez souvent partagé par le touriste mystique de type aventurier, à cette différence près que l'aventurier espère trouver le «vrai chamane " vivant. Aussi, ses premiers contacts avec les chamanes kyzyliens, trop urbanisés, sont-ils une source de déception. Cette impression pénible est vite surmontée par les visiteurs mystiques de type non aventurier, plus âgés, et satisfaits de ne pas avoir à entreprendre de longs et pénibles voyages dans les steppes de Touva à la recherche d'hypothétiques "vrais chamanes ", qui seraient de toute façon moins compréhensifs que ceux de Kyzyl.

\section{Le système des échanges dans le chamanisme kyzylien}

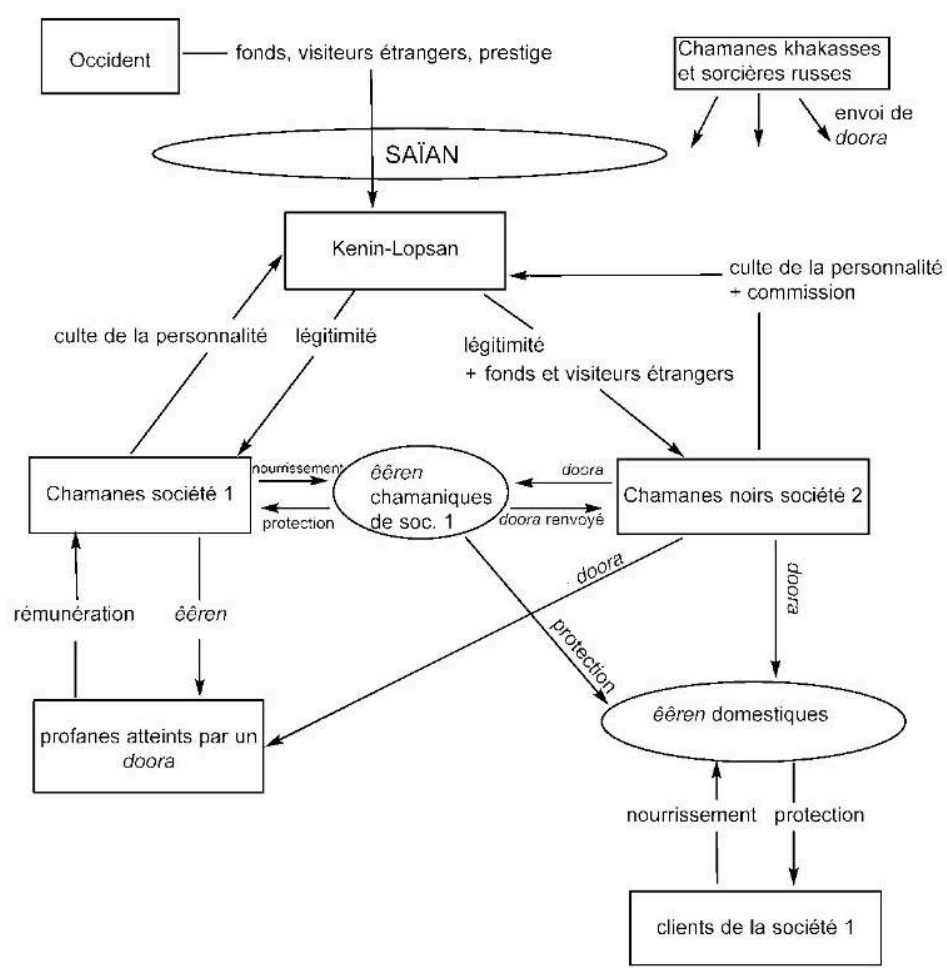

Pour le flux de doora le point de vue est celui de la société ।

( )n ne devient pas chamane à 'louva sans l'aveu de henin-J opsan, lequel se mérite par l'exécution d'un réritable culte de la personnalité. Discours dithỵtambiques, hommages dans des articles, inclinations profondes en public devant sa personne, triomphes organisés en son honneur, telles sont les marques d'allégeance nécessaires pour être reconnu comme «vtai chamane». I a pesition légitimante de Kenin-] dopsan lui vient ellemême d'« au-delà des Säan ", en raison du prestige que lui apportent les visites des ()ecidentaux et le tirre de "Trésor du chamanisme" dont ils l'ont honoré. Kenin-d opsán envole ses visiteurs et une partic de leurs subrentions à la société 2, qui en échange lui donne une commission sur les honoraires recus pour les stages chamaniques. lin compétition pour le drainage des étrangers, les différentes sociétés chamaniques entretiennent entre elles un cycle de dotor ou maurais sorts. Ce cycle est sans fin car les ferto chamaniques renresient les donz à leurs expéditeurs. I es doma tombés sur les profanes démunis sont une source de rémuncration pour les chamanes qui soignent ces victimes et leur fabriguent des êtef domestiques. Certains dorom sont absorbés pat

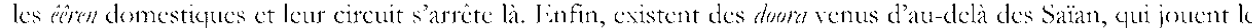
mème rôle que ceux de la société 2. I a circulation des dorfa devtait en principe s'estomper lorsque tous les foyers touvas seront pourvus d'êten, comme ils l'étaicnt avant la Révolution. Cependant les ërren vicillissent et meurent, ils perdent leur pouroir et laissent leurs maitres à la merci des doom. D'un point de vue sociologique il est à craindre qu'en cas de disparition de Kenin-l opsan, aujourd'hui fort ágé, le circuit intégrateur de la légitimation ne s'effondre, laissant toute place a une circulation inflationniste de dorm menée par des chamanes en guere ouverte, circulation imaginaire certes, mais aux effets réclement déléteres pour le lien social. 


\section{BIBLIOGRAPHIE}

Beffa, M.-L. et L. Delaby

1999 Festins d'âmes et robes d'esprits. Les objets chamaniques sibériens du Musée de l'Homme (Paris, Publications scientifiques du Muséum), 241 p. [Mémoires du Muséum national d'Histoire naturelle, 181].

Broše, P'er-Kristian (éd.)

Tuva (Moscou, Avangard), 159 p. [Le Pti Fjute].

Hoppal, M. 2003 Tracing shamans in Tuva, Acta Ethnographica Hungarica, XLVIII, 3-4, pp. 465-481.

Kenin-Lopsan, M. B.

2000 Tuvinskie šamany - znatoki tainctvennyx jazykov, in D. A Funk et V. I. Xaritonova (éd.), Êtnologičeskie issledovanija po šamanstvu i inym rannim verovanijam i praktikam, VII, 2 (Moscou, IÊA RAN), p. 225.

2002 Mify tuvinskix šamanov (Kyzyl, Novosty Tuvy), 543 p.

Naoumova, G.

2002 Taïga transes : voyage initiatique au pays des chamanes sibériens (Paris, Calmann-Lévy), 264 p.

Pimenova, K. I.

à paraitre The emergence of a new social identity. Trajectories and biographies of post-soviet shamans in the Republic of Tuva.

Vajnštejn, S. I.

1961 Tuvincy-todžency. Istoriko-êtnografičeskie očerki (Moscou, Izdatel'stvo vostočnoj literatury). 1991 Mir kočevnikov Centra Azii (Moscou, Nauka).

\section{NOTES}

1. Annexée en 1944 par l'URSS, Touva est aujourd'hui une république autonome de la Fédération de Russie. Sa population est de 310000 habitants dont 68 \% d'indigènes (2001).

2. Les êeren sont des objets chamaniques que l'on rencontre dans les yourtes, les appartements, et parmi l'attirail du chamane. Ils sont considérés comme des êtres vivants mais se distinguent des entités que l'ethnographie appelle communément «esprits» en raison de leur nature essentiellement matérielle.

3. Möngüš Baraxovič Kenin-Lopsan, né en 1930, cumule aujourd'hui dans la société kyzylienne les fonctions de savant et de chamane suprême. Sa grand-mère était chamane, et a été emprisonnée quinze ans pour cette raison. Après des études à l'université de Léningrad, il a obtenu le titre de docteur en histoire. Il est l'auteur de nombreux ouvrages sur le folklore touva, mais aussi de romans régionalistes. Fondateur de la société Düngür en 1992, il a reçu en 1994 pour son œuvre du Fonds pour les études chamaniques de Michael Harner le titre de «Trésor vivant du chamanisme ", cf. Pimenova (à paraître).

4. P'er-Kristian Broše éd. (2003, p. 66).

5. Pierre-Christian Brochet, l'auteur (français) du Petit Futé russe, détaille ainsi ses talents : «Elle fait des divinations, retire les énergies mauvaises, soigne les gens avec des massages chamaniques » (ibid.).

6. Le musée des Soixante-Héros, du nom de soixante insoumis qui prirent les armes contre les Mandchous en 1883. 
7. Beffa et Delaby (1999, p. 59 : cas de deux chamanes évenks chamanisant ensemble).

8. http://www.hyder.demon.co.uk/Kara-Ool.htm

\section{RÉSUMÉS}

Ces dix dernières années, la république de Touva a vu renaître en milieu urbain le chamanisme sous des formes institutionnelles radicalement neuves. Après une description des principales associations chamaniques, cet article présente les problèmes de concurrence et de légitimité liés à ces brutales évolutions.

During the last ten years, the Republic of Tuva has seen a rebirth of shamanism in urban areas in radically new institutional forms. After a description of the principal shamanist associations, this article presents the problems of competition and legitimacy connected to these brutal changes.

\section{INDEX}

Thèmes : chamanisme, changement, institutions

Mots-clés : chamanisme sibérien, post-socialisme

Index géographique : Russie, Touva

Keywords : post-socialism, siberian shamanism

nomsmotscles Touva

\section{AUTEUR}

\section{CHARLES STÉPANOFF}

Ancien élève de l'ENS, docteur de l'EPHE en Anthropologie religieuse (2007), maître de conférences à l'EPHE (2008). Auteur d'un film, Touva, république des chamanes 